\title{
Study of Liquid Lead Corrosion of Fast Nuclear Reactor and Its Mitigation by Using Molecular Dynamics Method
}

\author{
Artoto Arkundato, Zaki Su'ud, Mikrajuddin Abdullah, and Widayani Sutrisno
}

\begin{abstract}
Corrosion of iron in lead liquid coolant of fast nuclear reactor was studied using the molecular dynamics simulation method. The main goal of this work is to investigate effect of oxygen injection into lead coolant in order to reduce the corrosion rate of iron (major component of clad steel). By evaluating the mean square displacement curves, radial distribution function curves, iron diffusion coefficients and also observed the crystal structure of iron before and after oxygen injection then we concluded that the best value for significant, efficient and effective reduction of pure iron corrosion will be achieved by injection of $1.00 \%$ oxygen atoms (compared to the number of coolant atoms) into lead coolant, at temperature 750

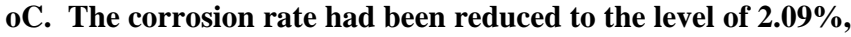
compared without oxygen injection.
\end{abstract}

Index Terms-Liquid lead corrosion, fast nuclear reactor, molecular dynamics method, oxygen contents.

\section{INTRODUCTION}

Liquid lead is a candidate coolant material for fast nuclear reactors design because it's favorable in chemical, physical and thermodynamics [1]. The lead coolant has a boiling temperature above the clad melting temperature, and low melting point. Lead is inert with respect to water. Lead may permit reduction of costs for operation \& maintenance of reactor. However, it is well known that the steel of reactor cladding are severely corroded if they are exposed to the pure lead/lead alloy coolant directly at medium to high temperatures [1]-[8]. The corrosion has limited the lifetime of reactor operation. This crucial problem has presented a critical challenge in application of lead coolant for fast reactor design. Then to handle the corrosion attack we need a way of reducing the corrosion rate or by creating and exploring novel materials [2], [6], not only under normal condition but also in the temporary anomalous condition especially for safety of heat transfer systems [5]. Meanwhile many experiments [2]-[8] about liquid lead/lead alloy corrosion have been reported, however a full understanding and how to manage corrosion are still not completed, yet. In addition it is not all experiments may or easy be done within an operated reactor. In this situation then theoretical studies

Manuscript received March 5, 2012; revised November 15, 2012. This work is a part of doctoral program research in physics at physics department, Institut Teknologi Bandung, Indonesia.

Artoto Arkundato is with the Physics Department, Jember University, Jember, Indonesia. (68121) (e-mail: a.arkundato@gmail.com).

Zaki Su'ud, Mikrajuddin Abdullah, and Widayani Sutrisno are with Physics Department, Institut Teknologi Bandung, Bandung, (40132), Indonesia. (40132) (e-mail: szaki@fi.itb.ac.id, din@fi.itb.ac.id, widayani@fi.itb.ac.id). have become a very important hint.

In this present work we study the corrosion by employing the molecular dynamics (MD) simulation method that is a powerful technique for material computation [9]-[10]. The application of MD simulation method to study the corrosion of liquid metal is relatively new and an interesting research topic. Alan et al had studied the penetration depth of iron atoms in liquid metal using MD simulation [11]. However they did not explore the thermodynamics properties of the corrosion and also did not study the corrosion reduction, yet. Authors had applied MD simulation method to calculate the diffusion coefficient of corrosion [12].

The purpose of the current research is to find a way of reducing the corrosion rate. We chose an oxygen atom to be injected into the liquid lead coolant. The use of oxygen for reducing the corrosion rate is an experimental hot topic [1]-[2], [7]-[8]. In our current research we want to predict the percentage of injected oxygen for the most significant and efficient reduction of corrosion rate. This research is also the improvement of our previous research [12], where we have used new Lennard-Jones (LJ) potential parameters as reported by Zhen and Davies [13] and also using new model of simulation. To evaluate the corrosion we observed and analyze the diffusion coefficient of iron (steel cladding) in hot liquid-lead environment.

\section{Simulation Details}

\section{A. Molecular Dynamics}

Molecular dynamics is a simulation technique in which the interacting atomic systems are allowed to evolve for a specified period of time, creating a trajectory. There are several schemes and also free MD codes to do molecular dynamics. In our work we used the Moldy code for simulating the atomic systems [9]. Moldy is a computer program for performing molecular dynamics simulations of condensed matter. Denoting $f_{i \alpha j \beta}$ is force exerted by atom $\alpha$ of molecule $i$ on atom $\beta$ of molecule $j$, total force acting on molecule $i$ is

$$
\overrightarrow{F_{i}}=\sum_{j} \sum_{\beta} \sum_{\alpha} \vec{f}_{i \alpha j \beta}
$$

The force determining the dynamics of the system may be derived from the specific potential function, $u_{i \alpha j \beta}\left(\vec{r}_{i \alpha j \beta}\right)$. The indices $i$ and $j$ run over all molecules in the system and $\alpha$ and $\beta$ over sites on the respective molecule. The total potential energy of the system is 


$$
U=\sum_{i} \sum_{j>i} \sum_{\alpha} \sum_{\beta} u_{i \alpha j \beta}\left(\vec{r}_{i \alpha j \beta}\right)
$$

where $\vec{f}_{i \alpha j \beta}=-\nabla u_{i \alpha j \beta}\left(\vec{r}_{i \alpha j \beta}\right)$ is the force acting on site $\beta$ of molecule $j$ from site $\alpha$ of molecule $i$. The (1) may be written as

$$
\vec{F}_{i}=-\nabla \sum_{j} \sum_{\beta} \sum_{\alpha} u_{i \alpha j \beta}\left(\vec{r}_{i \alpha j \beta}\right)
$$

Then the dynamics of the systems can be governed by the Newton-Euler equation of motion:

$$
M_{i} \frac{d^{2} \overrightarrow{R_{i}}}{d t^{2}}=\vec{F}_{i}
$$

where $\vec{R}_{i}=1 / M_{i \alpha} \sum m_{i \alpha} \vec{r}_{i \alpha}$ is the centre of mass of molecule $i$. The Newton-Euler equations can be solved numerically by using Modified Beeman algorithm [9]. The algorithm needs five steps to calculate the trajectories of the systems as below:

$$
\begin{aligned}
& ================================== \\
& x(t+\Delta t)=x(t)+\Delta t \dot{x}(t)+\frac{1}{6} \Delta t^{2}[4 \ddot{x}(t)-\ddot{x}(t-\Delta t)](\mathrm{i}) \\
& \dot{x}^{(p)}(t+\Delta t)=\dot{x}(t)+\frac{1}{2} \Delta t[3 \ddot{x}(t)-\ddot{x}(t-\Delta t)] \\
& \ddot{x}(t+\Delta t)=F\left(\left\{x_{i}(t+\Delta t), \dot{x}_{i}^{(p)}(t+\Delta t)\right\}, i=1 \ldots n\right) \\
& \dot{x}^{(c)}(t+\Delta t)=\dot{x}(t)+\frac{1}{6} \Delta t[2 \ddot{x}(t+\Delta t)+5 \ddot{x}(t)-\ddot{x}(t-\Delta t)]
\end{aligned}
$$

Replace $\dot{x}^{(p)}$ with $\dot{x}^{(c)}$, goto (iii), iterate to convergence (v)

The symbol $x$ represents any dynamic variable as the centre-of-mass coordinate $(R)$. The symbol $\dot{x}^{(p)}$ and $\dot{x}^{(c)}$ represent "predicted" and "corrected" velocity respectively. The predictor-corrector cycle of steps (ii) to (v) then is iterated until the predicted and corrected velocities have achieved a good convergence to a relative precision of better than 1 part in $10^{-7}$ [9].

\section{B. Interatomic Potentials}

In our work we used the LJ potential functions to describe the atomic interactions among atoms as

$$
u(r)=4 \varepsilon\left[(\sigma / r)^{12}-(\sigma / r)^{6}\right]
$$

where $\varepsilon$ and $\sigma$ are the LJ potential parameters. How did we establish the potential parameters for our corrosion problem? In our research we accommodated three kinds of atom: $\mathrm{Fe}$ (iron), $\mathrm{Pb}$ (lead) and $\mathrm{O}$ (oxygen) to simulate the corrosion reduction process. To maintain the validation of $\mathrm{LJ}$ potential we used the $\mathrm{Fe}-\mathrm{Fe}$ and $\mathrm{Pb}-\mathrm{Pb}$ pair interaction of metals based on the experimental data as reported by Zhen and Davies [13] For the $\mathrm{O}-\mathrm{O}$ interaction we used the data as reported by Lemmon and Jacobsen [14]. Whereas for cross-interaction: $\mathrm{Fe}-\mathrm{Pb}, \mathrm{Fe}-\mathrm{O}, \mathrm{Pb}-\mathrm{O}$ we approached the $\mathrm{LJ}$ parameters by using the popular Lorentz-Berthelot mixing rule [11]-[12], [15]:

$$
\begin{gathered}
\sigma_{A B}=\left(\sigma_{A A}+\sigma_{B B}\right) / 2 \\
\varepsilon_{A B}=\sqrt{\left(\varepsilon_{A A} \times \varepsilon_{B B}\right.}
\end{gathered}
$$

Table I summarizes the LJ potential parameters that used in our LJ-MD simulation to study corrosion phenomena.

TABLE I: THE LENNARD-JONES PARAMETERS

\begin{tabular}{lcclcc}
\hline \hline $\begin{array}{l}\text { Pair } \\
\text { Interaction }\end{array}$ & $\sigma(\AA)$ & $\varepsilon(\mathrm{eV})$ & $\begin{array}{l}\text { Pair } \\
\text { Interaction }\end{array}$ & $\sigma(\AA)$ & $\varepsilon(\mathrm{eV})$ \\
\hline $\mathrm{Fe}-\mathrm{Fe}$ & 0.4007 & 2.3193 & $\mathrm{Fe}-\mathrm{Pb}$ & 0.2766 & 2.7541 \\
$\mathrm{~Pb}-\mathrm{Pb}$ & 0.1910 & 3.1888 & $\mathrm{Fe}-\mathrm{O}$ & 0.0639 & 2.8737 \\
$\mathrm{O}-\mathrm{O}$ & 0.0102 & 3.4280 & $\mathrm{~Pb}-\mathrm{O}$ & 0.0441 & 3.3084 \\
\hline \hline
\end{tabular}

\section{Hot Corrosion Mechanism and Properties Calculation}

The corrosion of structural metals or alloys in liquid metal is for the most part due to the dissolving of the various constituents $(\mathrm{Fe}, \mathrm{Ni}, \mathrm{Cr}$, etc.) of the metals or alloys by the liquid metal. Corrosion may be understood as a degradation of structural materials (metals) into its constituent atoms. Generally the degradation follows the chemical or electrochemical action; for example is the rusting of iron. Thus, corrosion by this definition denotes the transfer of electrons that means there is a chemical reaction.

But this is not usually the case in liquid metal corrosion then to define the corrosion by destruction of a solid metal by a liquid metals the corrosion definition must be broadened to allow for the solution or solubility of a solid metal in a liquid metal wherein no transfer of electrons (chemical reaction) is involved [16]. Liquid metal corrosion for the most part simply depends on the solution rate and the extent of solubility of the solid metal in the liquid metal.

Temperature is one of the most important variables, because the higher the temperature the higher the solubility of the solid metal in the liquid metal. Also, as the temperature increases, diffusion rates increase, which is quite important in certain types of liquid metal corrosion. A very high temperature of operating reactor core (liquid lead) creates high temperature environment for steel cladding that in turn causes heavy corrosion. This is a non-galvanic hot corrosion.

Based on above consideration then we established that in our work the corrosion will be treated as a pure diffusion process without any chemical reaction. This seems to validate the use of classical MD simulation method for our corrosion study, primarily the iron corrosion in the high temperature stagnant coolant. Due to we consider the corrosion is the diffusion phenomena the in our research we need to calculate the diffusion coefficient of the metals. The diffusion coefficient can be calculated by finding the trajectory of all atoms that is the solution of Newton equation for corroded system. From the trajectories of Fe atoms after complete MD Moldy simulation then we will calculate the diffusion coefficient $D$ of iron Fe (major component of steel). The diffusion coefficient calculation needs the mean square displacement (MSD) calculation as a part of Einstein relation for $D$ coefficient. Then we also need to calculate the temperature dependence of diffusion coefficient $D(T)$ by employing the popular Arrhenius formula [12]. The three important equations are: 


$$
\begin{aligned}
M S D & =\left\langle|\vec{R}(t)-\vec{R}(0)|^{2}\right\rangle \\
D & =\lim _{t \rightarrow \infty} M S D / 6 t \\
D(T) & =D_{0} \exp (-A / \Re T)
\end{aligned}
$$

where $t$ is time, $T$ is temperature, $A$ is activation energy for corrosion to be happen and $\mathfrak{R}$ is gas constant. To know the structure of materials we also calculate the radial distribution function or $g(r)$. This calculation has been facilitated by Moldy code easily. To know the microscopic structure of material we will plot the coordinate xyz of atoms.

\section{Simulation Procedure}

The simulation procedure of corrosion and its reduction can be described as below steps:

1) Simulation of $\mathrm{Fe}$ in liquid $\mathrm{Pb}$ at several high temperatures: $600{ }^{\circ} \mathrm{C}-1100{ }^{\circ} \mathrm{C}$.

2) Calculate the diffusion coefficient $D(T)$ of Fe by using (10) and compare to available experimental data as reported by Zhang and $\mathrm{Li}$ [1]. This is to see the validation of simulation model.

3) Continue to simulate oxygen injection in $\mathrm{Fe}-\mathrm{Pb}$ system at $750{ }^{\circ} \mathrm{C}$, the possible high temperature of reactor. Inject oxygen atoms into coolant for several percentages: $0.5 \%$, $1.0 \%, 1.5 \%, 2 \%, 2.5 \%, 3 \%, 3.5$ and $4.0 \%$. Analyze the MSD, RDF and the microscopic structure of Fe material. Check at what percentage the oxygen injection will be significant and efficient for reducing corrosion.

4) Simulate the $\mathrm{Fe}-\mathrm{Pb}-\mathrm{O}$ system with fixed percentage of injected oxygen based on step (iii). Calculate the $\mathrm{Fe}$ diffusion coefficient, $D(T)$, after reduction.

\section{RESULTS AND DISCUSSION}

\section{A. Fe Diffusion Coefficient without Oxygen Injection}

First as the simulation procedure (i) we simulated $\mathrm{Fe}$ material in liquid lead coolant $(\mathrm{Fe}-\mathrm{Pb}$ simulation without oxygen injection). The simulation was prepared as a simulation box where the iron bulk $(1729 \mathrm{Fe}$ atoms in $9 \times 9 \times 9$ bcc lattice with lattice constant $a=2.83 \AA$ ) was in the center of the system covered by liquid lead coolant $(5577 \mathrm{~Pb}$ atoms with atomic density 0.0274 atoms $/ \AA^{3}$ ). The MD simulation box then was a super cell with $63.2 \times 63.2 \times 63.2 \AA^{3}$ dimension, as in Fig. 1.

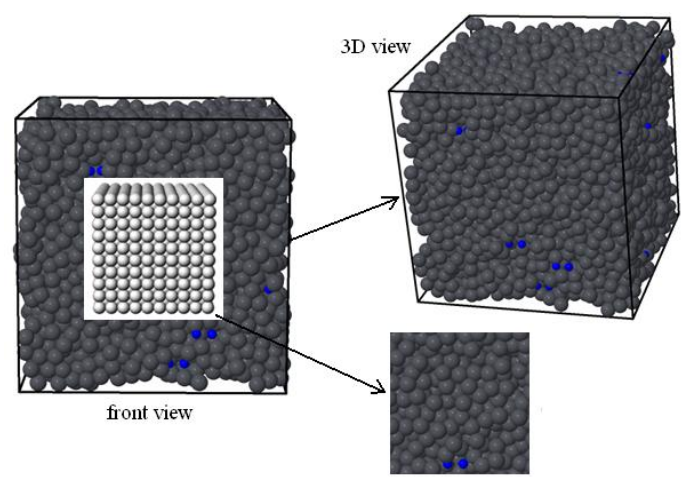

Fig. 1. The simulation box of the Fe-Pb-O system. The blue color is oxygen atom for configuration of next simulation (with Jmol at http://www.jmol.org)
Then we simulated the system for different elevated temperatures: $600{ }^{\circ} \mathrm{C}, 650{ }^{\circ} \mathrm{C}, 700{ }^{\circ} \mathrm{C}, 750{ }^{\circ} \mathrm{C}, 800{ }^{\circ} \mathrm{C}, 850{ }^{\circ} \mathrm{C}$, $900{ }^{\circ} \mathrm{C}, 950{ }^{\circ} \mathrm{C}, 1000{ }^{\circ} \mathrm{C}$, and $1050{ }^{\circ} \mathrm{C}$. To control the simulation in Moldy code, we used the NPT Anderson constant-pressure at nearly zero, Nose-Hoover thermostat, timesize of 0.0001 ps and 100000 steps of modified Beeman integration [9]. The properties average were done starting at step 19000 after equilibration.

After complete MD simulation then for every temperature we calculated all properties: $M S D, D$ and $D(T)$ by using (8)-(10). Fig. 2. is a plot of $\log D$ vs. $1 / T$ from Fe diffusion MD simulation data.

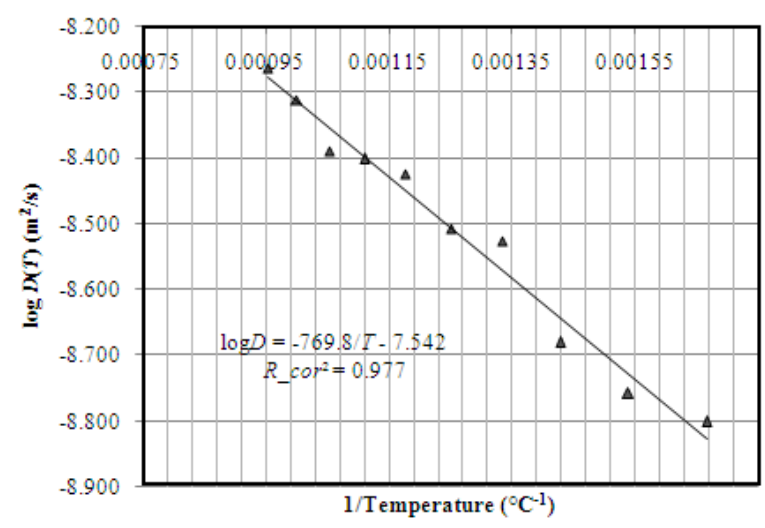

Fig. 2. Plot of $\log \mathrm{D}$ vs. 1/T of Fe diffusion.

The linear equation of the line in Fig. 2 has mathematical expression as

$$
\log D(T)=-769.814 / T-7.542
$$

We can see the graph is linearly enough with correlation coefficient $R_{\text {cor }}^{2}=0.977$. By using (10) and (11) then we can predict the temperature dependence of $\mathrm{Fe}$ diffusion is:

$$
D(T)=2.865 \times 10^{-8} \cdot \exp (-1764.971 / T)
$$

Now we calculate the coefficient diffusion by (12) for temperature $750^{\circ} \mathrm{C}$ :

$$
D\left(T=750{ }^{\circ} \mathrm{C}\right)=2.723 \times 10^{-9} \quad\left[\mathrm{~m}^{2} / \mathrm{s}\right]
$$

W. M. Robertson had reported the experimental results that the Fe diffusion in pure $\mathrm{Pb}$ was $\mathrm{D}_{\mathrm{Fe} \rightarrow \mathrm{Pb}}=2.80 \times 10^{-9} \mathrm{~m}^{2} / \mathrm{s}^{1}$ for temperature of experiment $750{ }^{\circ} \mathrm{C}$ [1]. Comparing our MD result (13) with Robertson's result then there is an agreement about 99.22\%. Further Balbauer-Celerier and Barbier (BCB) using a correlation for lead that developed by Robertson (R) proposed that the Fe diffusion coefficient in lead liquid could be approached by [1]:

$$
D_{F e \rightarrow P b}(T)=4.9 \times 10^{-7} \cdot \exp (\{-44100 \pm 6300\} / \Re T)(14)
$$

We can call (14) as the BCBR equation for Balbauer-Celerier-Barbier-Robertson. Following (14) then the Fe diffusion coefficient in pure liquid lead coolant is in the range of $(1.31-5.75) \times 10^{-9} \mathrm{~m}^{2} / \mathrm{s}$ at temperature $750{ }^{\circ} \mathrm{C}$. 
Our MD calculation (13) then is also in a good agreement to the BCBR result (14). Fig. 3. shows a comparison between our MD calculation (12) and the BCBR experimental result (14).

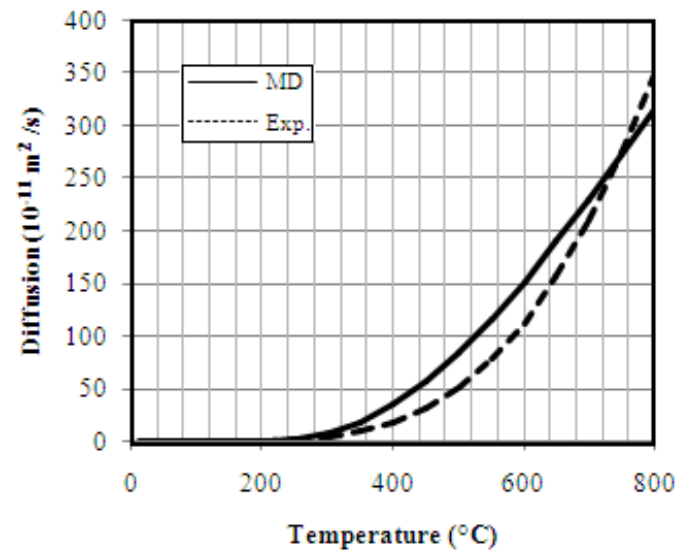

Fig. 3. The temperature dependence of Fe diffusion coefficient. Comparison between MD simulation (12) and BCBR equation (14).

From Fig. 3 we can see that our MD model of lennard-jones simulation may be applied to study liquid lead corrosion for temperatures about $750{ }^{\circ} \mathrm{C}$ downward. From this point we then can continue to investigate the corrosion reduction issue.

\section{B. Effect of Oxygen Injection}

The next simulation is to inject oxygen atoms to the lead coolant in order to reduce the corrosion rate. As above simulation the corrosion rate can be represented by evaluating the diffusion coefficient of iron. The simulation model is also as Fig. 1. but we had inserted and placed oxygen atoms into lead coolant evenly. We want to know at what percentage the oxygen atoms can reduce the corrosion rate significantly and efficiently. For this purpose we spread the oxygen atoms evenly into whole coolant with certain percentages (compared to the 5577 total quantity of $\mathrm{Pb}$ atoms of coolant): $0.00 \%, 0.50 \%, 0.75 \%, 1.00 \%, 1.50 \%, 2.00 \%$, $2.50 \%, 3.00 \%, 3.50 \%$ and $4.00 \%$. We simulated them at temperature $750{ }^{\circ} \mathrm{C}$ only, calculated diffusion coefficient of iron and saw the material structure of steels based on the thermodynamics properties as MSDs, RDF functions and also the coordinates xyz of iron atoms. Fig. 4. is the MSD plots of $\mathrm{Fe}$ diffusion as a result of oxygen injection at different percentages of atomic quantity.

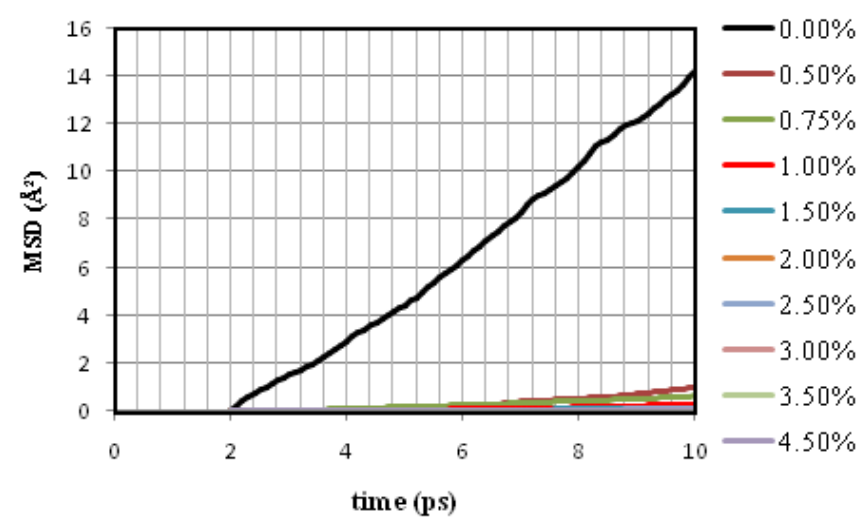

Fig. 4. The MSD curves of Fe diffusion by oxygen injection
We see from Fig. 4. that effect of oxygen injection can lower the MSD curve drastically. The top (black color) is the MSD curve of $\mathrm{Fe}$ diffusion without oxygen injection and describing the maximum corrosion rate at temperature 750 ${ }^{\circ} \mathrm{C}$.

The corrosion condition of Fe material can also be seen from the RDF functions, as in Fig. 5. For condition without oxygen injection the RDF curve (black color) shows a non-solid phase due to corrosion, whereas the injection of oxygen atoms will maintain the structure of Fe material in a solid phase condition. There are two groups with a coincide curves: the first group $(0.500 \%, 0.750 \%$ and $1.00 \%)$ and the second group $(1.50 \%-4.50 \%)$.

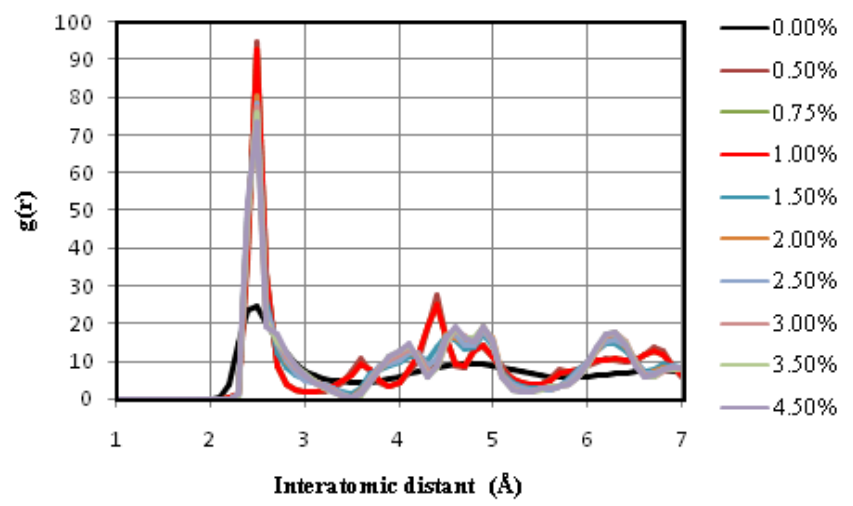

Fig. 5. The RDF curves of Fe diffusion at $750^{\circ} \mathrm{C}$

Now we arrive to the very important conclusion of simulation. By using (8) and (9) we made a plot of diffusion coefficient for every percentage. From Fig. 6. we can see that the most significant and efficient oxygen injection for reducing the corrosion rate is at the level of $1.00 \%$. More than $1.00 \%$ injection the diffusion coefficient curves show no more big change of reduction. Above $1.00 \%$ injection the curves show a stability of Fe diffusion. Below $1.00 \%$ injection the curves show drastic reduction of diffusion (corrosion). So we take a conclusion, based on Fig.4, Fig.5 and Fig. 6 that the best value of efficient oxygen injection for significant and effective corrosion reduction of steels is $1.00 \%$ oxygen atoms, at temperature $750{ }^{\circ} \mathrm{C}$.

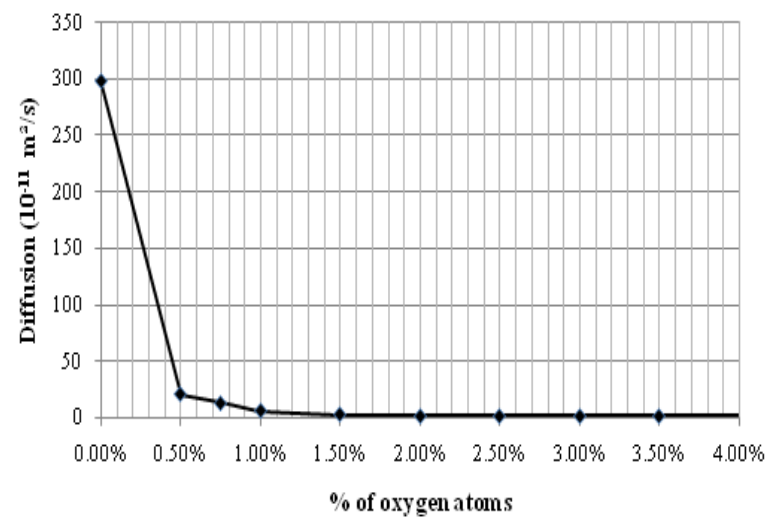

Fig. 6. The Fe diffusion coefficient for certain percentage of oxygen injection.

Fig. 7. shows a very attractive picture of a microscopic structure of Fe material that describing the effect of oxygen injection. Fig. 7(a) is the structure of Fe bcc crystal material 
before simulation. From Fig. 7(c) we can see that after $1.00 \%$ oxygen injection, the structure of Fe bcc material has been stabilized in solid phase whereas Fig.7(b) shows a big corrosion of $\mathrm{Fe}$ material without oxygen injection.

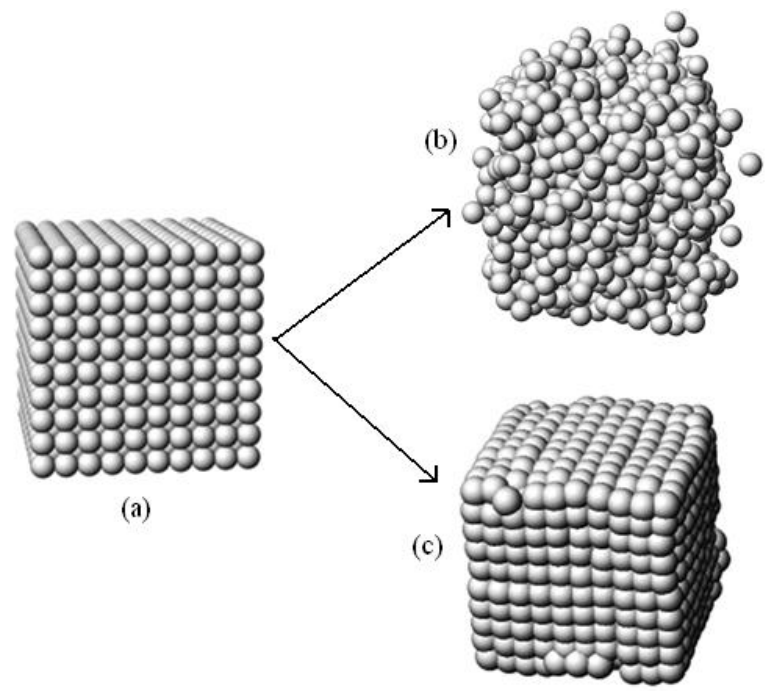

Fig. 7. The microscopic structure of Fe material before and after $1.00 \%$ oxygen injection to the liquid lead coolant (with Jmol at http://www.jmol.org).

\section{Fe Diffusion Coefficient with Oxygen Injection: Corrosion-Diffusion Reduction-Rate}

Now we calculate the temperature dependence of $\mathrm{Fe}$ diffusion coefficient with $1.00 \%$ oxygen injection. As previous simulation, we simulated the $\mathrm{Fe}-\mathrm{Pb}-(1.00 \% \mathrm{O})$ system for temperatures: $600{ }^{\circ} \mathrm{C}, 650{ }^{\circ} \mathrm{C}, 700{ }^{\circ} \mathrm{C}, 750{ }^{\circ} \mathrm{C}, 800$ ${ }^{\circ} \mathrm{C}, 850{ }^{\circ} \mathrm{C}, 900{ }^{\circ} \mathrm{C}, 950{ }^{\circ} \mathrm{C}, 1000{ }^{\circ} \mathrm{C}$, and $1050{ }^{\circ} \mathrm{C}$ with same control parameters of simulation. After complete simulation then we calculate the temperature dependence of Fe diffusion coefficient, by using (8)-(10). Fig. 8 shows the plot of $\log D_{1 \% \mathrm{O}}$ vs. $1 / T$. The line has a linear equation:

$$
\log D_{1 \% O}=-607.54 / T-9.437
$$

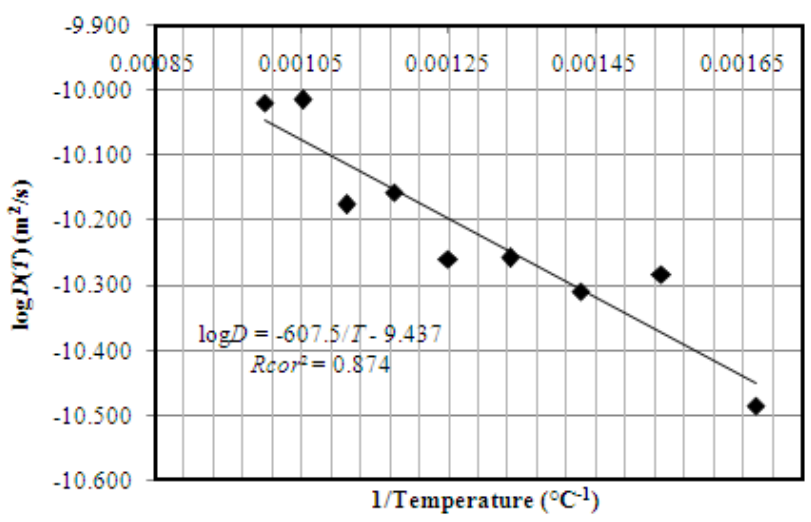

Fig. 8. Plot of $\log \mathrm{D} 1 \% \mathrm{O}$ vs. $1 / \mathrm{T}$ for Fe diffusion

By using (15) and (10) then we can determine the temperature dependence of Fe diffusion coefficient as:

$$
D_{1 \% O}(T)=3.653 \times 10^{-10} \cdot \exp (-1392.922 / T)
$$

Fig. 9 shows the Fe diffusion coefficient before and after $1.00 \%$ oxygen injection, comparing (12) and (16). We can calculate the diffusion rate at temperature $750{ }^{\circ} \mathrm{C}$ for (12) and (16). It is very interesting that the rate of $\mathrm{Fe}$ corrosion has been reduced to the level of $2.09 \%$ only, by injection of $1.00 \%$ oxygen atoms to lead coolant. This is very important prediction by MD calculation.

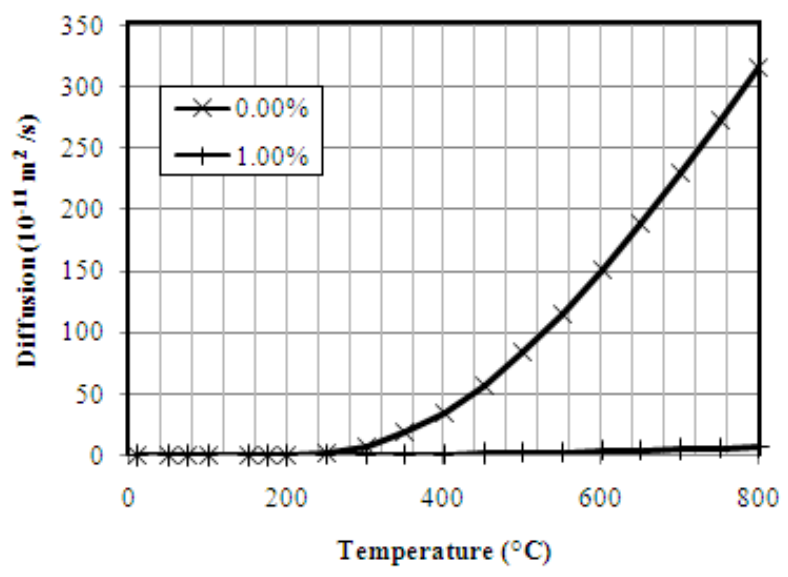

Fig. 9. Fe diffusion before oxygen injection (0.00\%) and after (1.00\%).

\section{Crystal Structure Analysis Using CNA Method}

In the above results, based on the Fig. 6 we have predicted that the effective reduction of iron corrosion is by injecting about $1.0 \%$ oxygen atoms into lead coolant. From Fig.7 we also see the injection of $1.0 \%$ oxygen has maintained the structure of iron in the solid phase. Now we analyze the structure of iron using CNA (Common Neighbor Analysis) method qualitatively. By using the CNA method we can calculate the number of crystal units (BCC, FCC, HCP or others). In this view we want to calculate how many $\mathrm{BCC}$ crystals in the iron material before and after oxygen injection. We want to see the stability of the iron material if we inject the oxygen atoms. Fig. 10 is the performance of iron structure for $0.0 \%$ and $1.0 \%$ of oxygen injection. The CNA calculation was done using OVITO code (http://ovito.org) [17]. We calculate the percentage of crystalline structure of iron i.e. the number of BCC crystal of iron, after oxygen injection compared with the initial 1729 BCC crystal (see sub-section III.A).

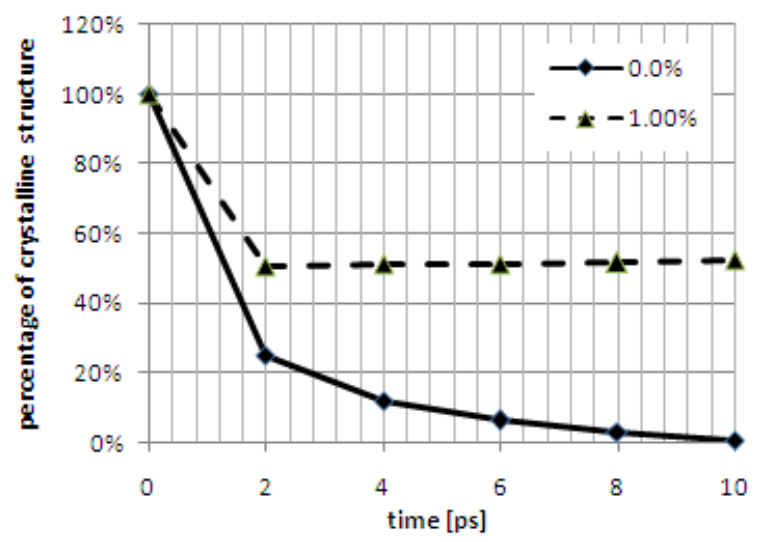

Fig. 10. The stability of crystal structure of iron in lead liquid

It is from Fiq.10 we can see that without oxygen injection 
then the structure of iron has experienced high corrosion. After 10 ps of simulation then the iron material has been in the non-crystalline structure. However if we inject about $1.0 \%$ oxygen atoms into lead coolant then the iron material structure has been stabilized in the crystalline form (about $50 \%$ ). By using the CNA method then we have shown that the injection of oxygen has stabilized the iron material in the form of crystalline phase.

\section{E. Corrosion Rate}

In the heat transfer system of nuclear reactor, the corrosion rate is function of many variables, depend on the system setup. Corrosion rate $R_{m}$ may be assumed to follow the relation [18]:

$$
R_{m}=k_{m}\left(c_{s}-c_{b}\right) \quad[\mu \mathrm{m} / \mathrm{yr}]
$$

where $k_{m}$.is a corrosion rate constant, that may be written analytically as [18]:

$$
k_{m}=\frac{3}{2 \Gamma(1 / 3)}\left(\frac{3}{1740}\right) v^{-2 / 3} D^{2 / 3} V(\lambda / 2)^{1 / 2}
$$

where $v$ is kinematic viscosity, $V_{f}$ is speed of flowing fluid, $\lambda$ is Fanning friction factor, and $D$ is iron diffusion coefficient in liquid lead. Diffusion coefficient $D$ is the one that is calculated in our simulation work. The Gamma number $\Gamma(1 / 3)$ is 2.6789 and $\lambda$ may use the Blasius correlation in turbulence current $\lambda=0.0791 \mathrm{Re}^{-0.25}$. If $d$ is the hydrolic diameter than $k_{m}$ may be simplified as:

$$
k_{m}=0.0133 v^{-0,542} D^{0,667} V^{0,875} d^{-0,125}
$$

Then based on the equations $(12,15,17-19)$ we can predicts the corrosion rate of iron in the case of flowing liquid lead. The data of $v, d, V$ can be measured experimentally, whereas the data of $D$ can be calculated and approached by Molecular Dynamics method. So in this research the diffusion coefficients have been calculated by molecular dynamics method and has been verified and compared with experimental results (12), whereas the corrosion rates can be calculated by (19) that is a function of diffusion coefficient D.

\section{SugGestions}

It is for the next research we will make further improvements for better understanding of corrosion: doing a large-scale LJ MD simulation, using more atoms, other better potential function, other element for injection and other liquid metals.

\section{REFERENCES}

[1] J. Zhang and N. Li, "Review of the studies on fundamental issues in LBE corrosion," Journal of Nuclear Materials, vol. 373, pp. 351-377, 2008.

[2] G. K. Zelenskii, A. G. Ioltukhovskii, M. V. L. Smirnova, I. A. Naumenko, and S. A. Tolkachenko, "Corrosion resistance of fuel element steel cladding in a lead coolant," Metal Science and Heat Treatment, vol. 49, no. 11 - 12, pp. 533-538, 2007.
[3] A. Maitre, M. Francois, R. Podor, and J. C. Gachon, "Approach of the corrosion behaviour of T91 steel in lead or lead-bismuth liquid bath," Material Science Forum, vol. 461-464, pp. 1141-1148, 2004.

[4] D. Sapundjiev, S. V. Dyck, and W. Bogaerts, "Liquid metal corrosion of T91 and A316L materials in Pb-Bi eutectic at temperatures 400-600 ${ }^{\circ}$ C," Corrosion Science, vol. 48, pp. 577-594, 2006.

[5] A. Z. Kashezhev, M. Kh. Ponegev, V. A. Sozaev, A. I. Khasanov, and A. G. Mozgovo, "An experimental investigation of the wetting of reactor steels with molten lead and bismuth," High Temperature, vol. 48, pp. 756-758, 2010.

[6] A. K. Rivai and M. Takahashi, "Corrosion characteristics of materials in $\mathrm{Pb}-\mathrm{Bi}$ under transient temperature conditions," Journal of Nuclear Materials, vol. 398, pp. 139-145, 2010.

[7] V. Zhang, P. Hosemann, and S. Maloy, "Models of liquid metal corrosion," Journal of Nuclear Materials, vol. 404, pp. 82-96, 2010 .

[8] C. Fazio, I. Ricapito, G. Scaddozzo, and G. Benamati, "Corrosion behaviour of steels and refractory metals and tensile features of steels exposed to flowing $\mathrm{PbBi}$ in the LECOR loop," Journal of Nuclear Materials, vol. 318, pp. 325-332, 2003.

[9] K. Refson, "Moldy: a portable molecular dynamics simulation program for successive and parallel computers," Computer Physics Communications, vol. 126, no. 3, pp. 309-328, April 2000.

[10] G. J. Auckland, K. D. Mellow, S. L. Daraszewicz, D. J. Hepburn, M. Uhrin, and K. Stratford, "The MOLDY short-range molecular dynamics package, “ Computer Physics Communications, vol. 182, pp. 2587-2604, July 2011.

[11] A. Maulana, Z. Su'ud, K. D. Hermawan, and Khairurrijal, "Simulation study of steels corrosion phenomenon in liquid lead-bismuth cooled reactors using molecular dynamics methods," Progress in Nuclear Energy, vol. 50, no. 2-6, pp. 616-620, March-August 2008.

[12] A. Arkundato, Z. Su'ud, and M. Abdullah, "Corrosion study of Fe in a stagnant liquid $\mathrm{Pb}$ by molecular dynamics methods," AIP Conference Proceeding, vol. 1244, New York, June 2010, pp. 136-144.

[13] S. Zhen and G. J. Davies, "L-J n-m potential energy parameters," phys.stat.sol.(a), vol. 78, pp. 595-605, 1983

[14] E. W. Lemmon and R. T. Jacobsen, "Viscosity and thermal conductivity equations for nitrogen, oxygen, argon and air," International Journal of Thermophysics, vol. 25, no. 1, pp. 21-69, 2004.

[15] J. Davoodi and M. J. Moradi, "Numerical Simulation of the Elastic Properties of Rh-20at\%Pd Nanowire," International Journal of Applied Physics and Mathematics, vol. 2, no. 1, January 2012.

[16] W. D. Manly. Fundamentals of liquid metal corrosion. Corrosion, 1959. [Online]. http://www.ornl.gov/info/reports/1956/3445603500959.pdf.

[17] A. Stukowski, "Visualization and analysis of atomistic simulation data with OVITO - the Open Visualization Tool," Modelling Simul. Mater. Sci. Eng, vol. 18, 2010.

[18] M. Machut, "Corrosion Behaviour of Steels for Lead-Alloy Cooled Fast Reactors and The Effect of Surface Modifications on Corrosion Performance,” Master Thesis, University of Wiscosin-Madison, 2007.

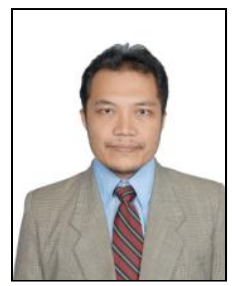

Artoto Arkundato was born on December 25, 1969 in Blitar, Indonesia. He got diploma in (theoretical) physics from physics department, Gadjah Mada University, Indonesia, and magister in (computational) physics from physics department, Institut Teknologi Bandung, Indonesia. Currently he continues the study at doctoral program, physics department, Institut Teknologi Bandung, Indonesia. His fields of research interest are computational physics and theoretical physics. In July 2006 he followed the international workshop on Electronics Structure Methods and Their Applications, JNCASR, Bangalore, India by financial support of ICTP Trieste, Italy. Now he is investigating the corrosion phenomena of nuclear reactor materials and its mitigation by using the molecular dynamics method. His scientific work had been published at AIP 2010 proceedings in conjunction with the ICANSE (International Conference on Advances in Nuclear Science and Engineering, 2009 international conference) and AIP 2012 proceeding in conjunction with the ICPAP (international conference on physics and its application) 2011. 


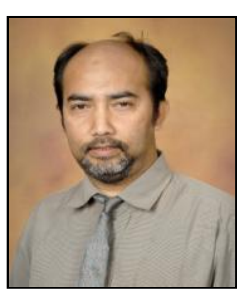

Zaki Su'ud received his Doctorate degree from nuclear engineering, Tokyo Institute of Technology, Japan. He is professor of physics, the head of nuclear physics laboratory and biophysics division at physics department, Institut Teknologi Bandung, Indonesia. His fields of research interest are nuclear reactor design and safety, nuclear reactor analysis code/program development/computer simulation, and nuclear instrumentation and control. His scientific works had been published/presented at many reputable international journals, proceedings, and international conferences. He was a chairman of ICANSE (International Conference on Advances in Nuclear Science and Engineering) conferences for three years $(2007,2009,2011)$.

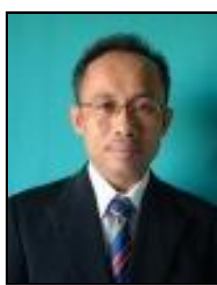

Mikrajuddin Abdullah received his Doctorate degree from chemical engineering, Hiroshima University, Japan (2002). He got postdoctoral fellow from Japan Society for the Promotion of Science, Japan (2002-2004). He is professor of physics at physics department, Institut Teknologi Bandung, Indonesia. His fields of research interest are sythesis of nanomaterials (nanoparticles, carbon nanutuber, nanocomposites), and applications of nanostructured materials (display, luminescent nano-ink, nanocatalyst, cosmetics, etc). Other interests in Physical Education (writting some books of physics for secondary and higher schools development of simple apparatus for physical demonstrations, etc.) His scientific works had been published/presented at many reputable international journals, proceedings, and international conferences.

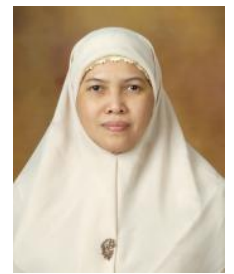

Widayani Sutrisno received her Doctorate degree from UMIST, Manchester, England. She was the head of post-graduate physics program (2009-2012), physics department, Institut Teknologi Bandung, Indonesia. Her fields of research interest are polymer material and its applications. Her scientific works had been published at many reputable international journals, proceedings, and international conferences 